\title{
Two Cases of Multiple Epidermolytic Acanthomas Mimicking Verruca Vulgaris
}

\author{
Hyun Jeong Byun, Donghwi Jang, Jongeun Lee, Se Jin Oh, Ji-Hye Park, Dong-Youn Lee \\ Department of Dermatology, Samsung Medical Center, Sungkyunkwan University School of Medicine, Seoul, Korea
}

Epidermolytic acanthoma (EA) is a rare benign tumor, which usually appears as a solitary small papule. However, there are a few case reports of multiple EA, most of which occurs on the genital area. Cases of multiple EA may mimic verruca vulgaris, condyloma accuminatum, seborrheic keratosis, and bowenoid papulosis, and therefore, can be easily misdiagnosed. A 78-year-old male presented with a 2-week history of discrete, small skin-colored papules around the anus. The other case involved a 47-year-old male with a 5 -year history of skin-colored papules on the scrotum. Skin biopsy of both cases revealed a well-demarcated papular lesion characterized by compact hyperkeratosis, perinuclear vacuolization, and reticular degeneration in the granular and upper spinous layer with coarse basophilic keratohyalin granules. Epidermal invagination was consistent with a cup-shaped type of EA. Both cases tested negative for human papillomavirus. We report typical cases of multiple EA, which should be considered as the differential diagnosis of small skin-colored papules in the anogenital area, to prevent the misdiagnosis. (Ann Dermatol 32(6) 512 515, 2020)

\section{-Keywords-}

Epidermolytic hyperkeratosis

Received September 19, 2019, Revised October 17, 2019, Accepted for publication October 23, 2019

Corresponding author: Dong-Youn Lee, Department of Dermatology, Samsung Medical Center, Sungkyunkwan University School of Medicine, 81 Irwon-ro, Gangnam-gu, Seoul 06351, Korea. Tel: 82-2-3410-3543, Fax: 82-2-34103869, E-mail: dylee@skku.edu ORCID: https://orcid.org/0000-0003-0765-9812

This is an Open Access article distributed under the terms of the Creative Commons Attribution Non-Commercial License (http://creativecommons. org/licenses/by-nc/4.0) which permits unrestricted non-commercial use, distribution, and reproduction in any medium, provided the original work is properly cited.

Copyright (c) The Korean Dermatological Association and The Korean Society for Investigative Dermatology

\section{INTRODUCTION}

Epidermolytic acanthoma (EA) is a rare benign acquired tumor with a wart-shaped surface. It usually appears as an asymptomatic brown papule measuring less than $1 \mathrm{~cm}$, and is known to occur frequently in middle-aged individuals ${ }^{1}$. EA can appear as a solitary or multiple papules, or very rarely as a disseminated form ${ }^{2}$. According to previous reports, 122 out of 131 cases were solitary EAs, and multiple EAs were much less frequent involving only 7 cases $^{2}$. EA is characterized by epidermolytic hyperkeratosis, which is also observed in bullous ichthyosiform erythroderma, Vörner's palmoplantar keratoderma, and linear epidermal nevi variants ${ }^{3}$. Clinically, it can be misdiagnosed as verruca vulgaris, condyloma accuminatum, seborrheic keratosis, and bowenoid papulosis ${ }^{2,4}$. In a survey of dermatopathologists conducted by the American Society of Dermatopathology, only $37 \%$ of dermatopathologists correctly diagnosed $\mathrm{EA}$, while another $37 \%$ misdiagnosed it as verruca vulgaris. Therefore, it is definitely a disease that requires attention for accurate diagnosis ${ }^{2}$.

\section{CASE REPORT}

A 78-year-old male presented with a 2-week history of papular lesions around the anus. Physical examination revealed multiple, flesh-colored papules measuring less than $1 \mathrm{~cm}$, without any symptoms such as pruritus or pain (Fig. 1A). The patient had a history of hypertension, glaucoma and urinary tract obstruction. Under polarized contact dermoscopy, hyperkeratotic callus like structure was observed without characteristic features of warts (Fig. 1B). Skin punch biopsy and human papillomavirus (HPV) staining were performed for accurate diagnosis. Examination under the low-power field revealed compact orthokeratotic hyperkeratosis. Invagination of the epidermis and fo- 

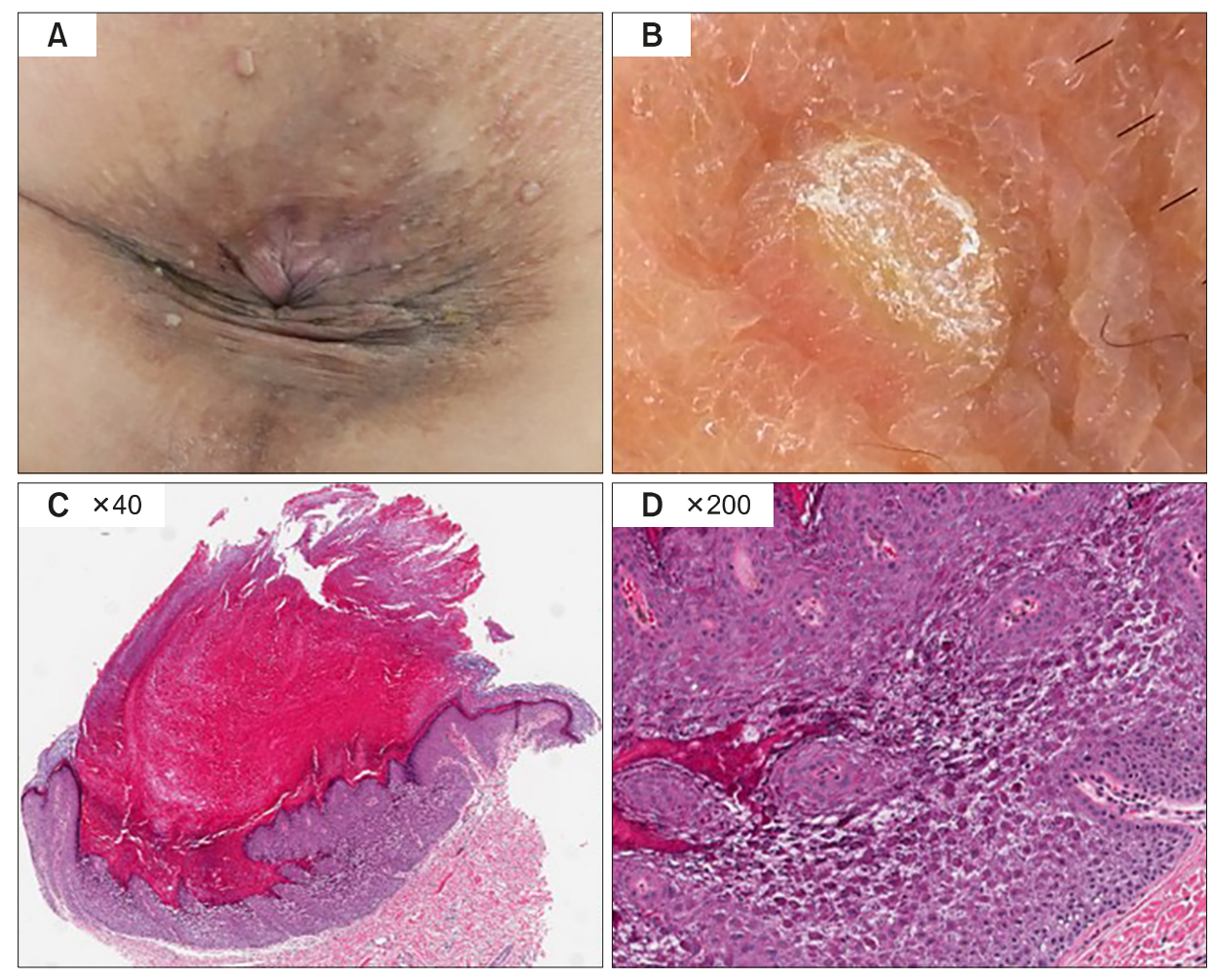

Fig. 1. (A) Multiple skin-colored papules located around the anus. (B) Hyperkeratotic callus like structure was observed without characteristic features of warts. (C) Epidermal invagination with compact hyperkeratosis $(H \& E, \times 40)$. (D) Reticular degeneration and perinuclear vacuolization with coarse basophilic keratohyaline granules (H\&E, $\times 200$ ). We received the patient's consent form about publishing all photographic materials.
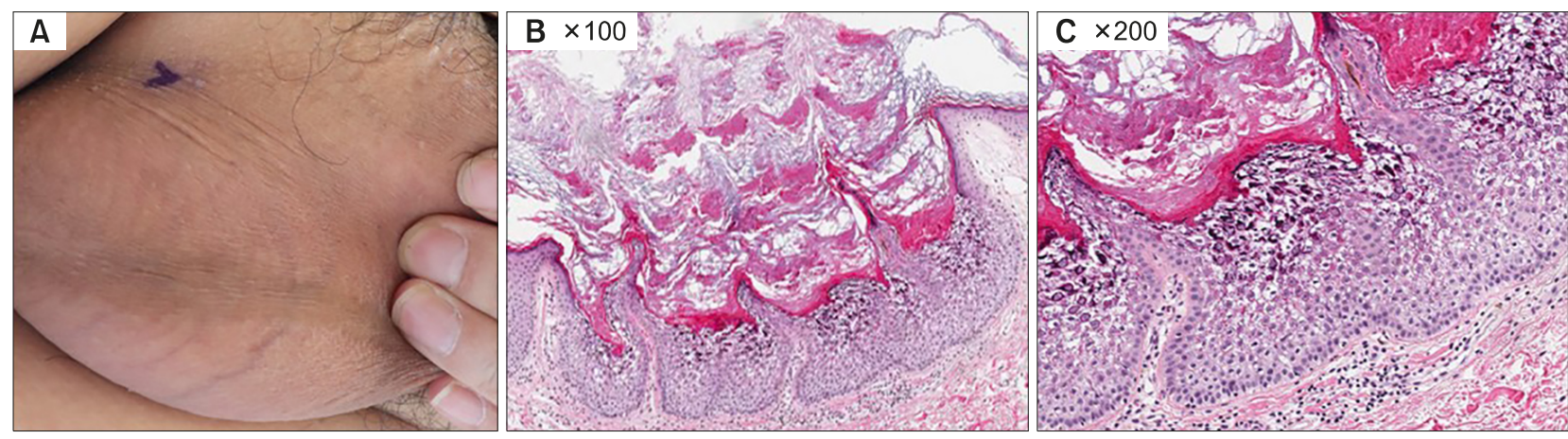

Fig. 2. (A) Multiple skin-colored papules located on the scrotum. (B) Cup-shaped epidermal invagination with hyperkeratosis (H\&E, $\times 100$ ). (C) Reticular degeneration and perinuclear vacuolization in the granular and upper spinous layers $(H \& E, \times 200)$.

cal papillomatous changes were observed (Fig. 1C). Under high-power field, clear spaces were detected around the nucleus, along with eosinophilic cytoplasm of keratinocytes in the spinous and granular layers of the epidermis. It is also possible to observe larger keratohyaline granules and reticular degeneration, which involved intercellular and intracellular edema in the epidermis (Fig. 1D). The patient tested negative for HPV, and was diagnosed with an EA with typical pathologic findings. The patient was monitored for 6 months without any further treatment after the skin biopsy, and no change in the lesion was observed after 6 months.

Another 47-year-old male presented with a 5-year history of skin lesions on the scrotum. A number of $2 \mathrm{~mm}$-sized flesh-colored papules were detected on the scrotum, and the patient complained of a slight pruritus (Fig. 2A). The patient had chronic hepatitis, and presented with onychomycosis of toenails, with no significant findings except for positive test results with rapid plasma reagin (RPR) in serum tests. Punch biopsy and HPV stain were performed for diagnosis. Under a low-power field, the epidermis was invaginated into a cup-shaped lesion, and hyperkeratosis was observed (Fig. 2B). Under a high power, reticular degeneration of epidermis was observed with vacuolar changes around the nucleus of keratinocytes (Fig. 2C). In addition, perivascular lymphocytic infiltration was seen in 
the superficial dermis. The patient tested negative for HPV. The second case was also diagnosed with EA based on clinical and pathological findings. A number of lesions on the scrotum were surgically removed in this patient.

\section{DISCUSSION}

According to a single institutional study reported previously, the incidence rates of EA were 9.08 per 100,000 specimens per year, with a declining trend ${ }^{2}$. The incidence rates of extragenital EA were higher than those of genital lesions, although multiple EA predominantly occurred in genital area $^{2}$.

Since EA is clinically prone to misdiagnosis into other lesions, further examinations such as dermoscopy or skin biopsy are needed for confirmation. In the previous report, dermoscopic findings of EA demonstrated pearly white areas, a cerebriform pattern, irregular pigmented grooves, and peripheral pigmented radial streak like areas ${ }^{5}$. However the lesion shown in the previous report clinically appeared similar to seborrheic keratosis, which resulted in different dermoscopic features with the present case. According to the report, "pearly white areas" were the striking feature of the EA, which corresponded to the compact hyperkeratosis above the hypergranulosis and acanthosis ${ }^{5}$. This finding was distinguishable with the dermoscopic findings of seborrheic keratosis which was characterized by comedo-like opening, milia-like cyst ${ }^{6}$, and that of verruca vulgaris which showed frogspawn patterns and lopped or dotted vessels within whitish papillae ${ }^{7}$. Other differential diagnosis such as genital warts, exhibit a white reticular network with the dermoscopy, and molluscum contagiosum displays polylobular amorphous structures surrounded by blurred telangiectasia ${ }^{7}$. The dermoscopic photograph of the present case did not show any features of seborrheic keratosis, verruca vulgaris, genital wart and molluscum contagiosum.

Even though dermoscopy can rule out some diagnosis, skin punch biopsies are more accurate tool for diagnosis. Characteristic histologic features include hyperkeratosis with reticular degeneration in the granular and spinous layer, also known as epidermolytic hyperkeratosis, perinuclear vacuolization, and larger keratohyaline granules ${ }^{8}$. The epidermolytic keratinocytes of EA are likely to be confused with the koilocytes of verruca vulgaris, one of the important differential diagnosis. Koilocytes have pyknotic nuclei surrounded by a clear halo, and mainly exist in the upper layer of the epidermis ${ }^{9,10}$. On the other hand, the epidermolytic keratinocytes have keratohyaline granules that are coarser than normal, and exist throughout the stratum malpighii ${ }^{11}$. Based on histopathology, EA can be div- ided into papillomatous, cup-shaped, and acanthotic types. In papillomatous type, hyperkeratosis and focal parakeratosis are observed along with papillomatous epidermal hyperplasia and hypergranulosis ${ }^{3}$. Cup-shaped lesions can be seen with well-demarcated epidermal invagination, acanthosis, and hyperkeratosis with focal parakeratosis ${ }^{3}$. The combination of papillomatous and cup-shaped lesions of EA have also been reported ${ }^{3}$. Acanthotic lesion involves only epidermal hyperplasia without papillomatous changes or epidermal invagination ${ }^{3}$. Inflammation usually appears as perivascular lymphocytic infiltration involving the superficial layer ${ }^{3}$.

The pathophysiology of EA is not clear. The possible etiological factors include ultraviolet irradiation, immunosuppression, trauma, viral infection, and mutations involving keratin 1 and 10 genes $^{2,12}$. Although a single reported case of EA tested positive for $\mathrm{HPV}^{13}$, it is possible that it may have been discovered accidentally, and many other reported EA cases do not appear to be related to HPV ${ }^{14,15}$. Both two cases reported here tested negative for HPV and were not in an immunosuppressed state. Also the locations involved in the present cases were anus and scrotum, which were not exposed to the ultraviolet radiation. Therefore, the lesions may have been caused by viral infection, trauma, or mutations in the Keratin 1 and 10 genes. Keratin 1 and 10 genes are thought to be associated with EA, because mutations in these genes result in dermatoses such as epidermolytic ichthyoses associated with hyperkeratosis and a similar histological pattern with $\mathrm{EA}^{2}$. Cohen et al. $^{12}$ found that immunohistochemical expression of keratin 1 and 10 genes was decreased in EA in the affected granular layer. However, another study conducted by Egozi-Reinman et al. ${ }^{16}$, found no mutations involving keratin 1 and 10 genes in EA lesions. No further genetic tests have been conducted in the present case. A previous report suggested that EA may have been triggered by wearing tight clothes around the waist ${ }^{17}$, which is a possible causative factor in our cases.

EA is a benign, non-contagious disease that is amenable to cryotherapy, $\mathrm{CO}_{2}$ laser treatment, or topical medications such as imiquimod, calcipotriol, tacrolimus and pimecrolimus ${ }^{18-20}$. It must be distinguished from contagious diseases such as verruca vulgaris and condyloma that require treatment, since treatment is not essential for $\mathrm{EA}^{8}$.

\section{CONFLICTS OF INTEREST}

The authors have nothing to disclose. 


\section{FUNDING SOURCE}

None.

\section{DATA SHARING STATEMENT}

Research data are not shared.

\section{ORCID}

Hyun Jeong Byun, https://orcid.org/0000-0002-4354-5655

Donghwi Jang, https://orcid.org/0000-0002-3495-4772

Jongeun Lee, https://orcid.org/0000-0002-1999-9948

Se Jin Oh, https://orcid.org/0000-0001-7525-4740

Ji-Hye Park, https://orcid.org/0000-0002-6699-5202

Dong-Youn Lee, https://orcid.org/0000-0003-0765-9812

\section{REFERENCES}

1. Yang JH, Kim JK, Won $\mathrm{CH}$, Chang SE, Lee MW, Choi JH, et al. Isolated epidermolytic acanthoma in a renal transplant recipient. Ann Dermatol 2011;23:415-416.

2. Roy SF, Ghazawi FM, Choate KA, MCNiff JM. Solitary and multiple epidermolytic acanthoma: a demographic and clinical study of 131 cases. J Cutan Pathol 2019;46:305-309.

3. Abbas $\mathrm{O}$, Wieland $\mathrm{CN}$, Goldberg LJ. Solitary epidermolytic acanthoma: a clinical and histopathological study. J Eur Acad Dermatol Venereol 2011;25:175-180.

4. Fletcher JW, Ramamurthi A, Parekh P. Presentation of epidermolytic acanthomas as multiple tan papules on the vulva. Proc (Bayl Univ Med Cent) 2016;29:198-199.

5. Behera B, Gochhait D, Sridivya P, Chandana S, Thappa DM, Malathi M. Dermoscopy of a solitary verrucous plaque on the back. J Am Acad Dermatol 2017;77:e37-e39.

6. Braun RP, Rabinovitz HS, Krischer J, Kreusch J, Oliviero M, Naldi L, et al. Dermoscopy of pigmented seborrheic keratosis: a morphological study. Arch Dermatol 2002;138:15561560.

7. Zalaudek I, Giacomel J, Cabo H, Di Stefani A, Ferrara G, Hofmann-Wellenhof R, et al. Entodermoscopy: a new tool for diagnosing skin infections and infestations. Dermatology 2008;216:14-23.

8. Lee TJ, Wu YH. Multiple epidermolytic acanthomas mimicking condyloma: a retrospective study of 8 cases. Int J Dermatol 2018;57:28-33.

9. Grayson W. Infectious disease of the skin. In: Calonje JE, Brenn T, Lazar AJ, McKee PH, editors. McKee's pathology of the skin. 4th ed. Edinburgh: Elsevier, 2012:760-895.

10. Xu X, Yun SJ, Erikson L, Chen L. Diseases caused by viruses. In: Elder DE, editor. Lever's histopathology of the skin. 11th ed. Philadelphia: Wolters Kluwer, 2015:781-815.

11. Shapiro L, Baraf CS. Isolated epidermolytic acanthoma. A solitary tumor showing granular degeneration. Arch Dermatol 1970; 101:220-223.

12. Cohen PR, Ulmer R, Theriault A, Leigh IM, Duvic M. Epidermolytic acanthomas: clinical characteristics and immunohistochemical features. Am J Dermatopathol 1997;19:232-241.

13. Jung JM, Lee $\mathrm{SH}$, Won $\mathrm{CH}$, Chang $\mathrm{SE}$, Lee $\mathrm{MW}$, Choi JH, et al. A case of multiple epidermolytic acanthoma of the scrotum: is the human papillomavirus a culprit? Ann Dermatol 2015;27:633-634.

14. Leonardi C, Zhu W, Kinsey W, Penneys NS. Epidermolytic acanthoma does not contain human papillomavirus DNA. J Cutan Pathol 1991;18:103-105.

15. Kazlouskaya V, Lambe J, Elston D. Solitary epidermolytic acanthoma. J Cutan Pathol 2013;40:701-707.

16. Egozi-Reinman E, Avitan-Hersh E, Barzilai A, Indelman $M$, Bergman R. Epidermolytic acanthoma of the genitalia does not show mutations in KRT1 or KRT10. Am J Dermatopathol 2016;38:164-165.

17. Sánchez-Carpintero I, España A, Idoate MA. Disseminated epidermolytic acanthoma probably related to trauma. $\mathrm{Br} \mathrm{J}$ Dermatol 1999;141:728-730.

18. Kukreja T, Krunic A. Multiple epidermolytic acanthomas must not be confused with genital human papillomavirus infection. Acta Derm Venereol 2009;89:169-171.

19. Jang BS, Jang HS, Park HJ, Kim MB, Oh CK, Kwon KS, Multiple scrotal epidermolytic acanthomas successfully treated with topical imiquimod. J Dermatol 2007;34:267-269.

20. Moulonguet I, Serre M, Herskovitch D. [Multiple epidermolytic acanthomas of the genitalia]. Ann Dermatol Venereol 2017;144:295-300. French. 